\title{
Concepciones sobre el Aprendizaje de Estudiantes de Pedagogía de la Universidad de Magallanes y Docentes en Ejercicio en la Educación Básica de la ciudad de Punta Arenas, Chile
}

\author{
Rodrigo A. Cárcamo ${ }^{(1)}$ y Pablo J. Castro(2) \\ (1) Univ. de Magallanes, Escuela de Psicología, Casilla 113-D, Punta Arenas-Chile \\ (2) Univ. de La Serena, Fac. de Humanidades, Dpto. de Psicología, Av. Matta 147, Coquimbo-Chile \\ (e-mail: rodrigo.carcamo@umag.cl; pablocastro@userena.cl)
}

Recibido Nov. 24, 2014; Aceptado Ene. 13, 2015; Versión final Abr. 17, 2015, Publicado Oct. 2015

\begin{abstract}
Resumen
Este estudio tuvo como objetivo conocer y analizar las concepciones acerca del aprendizaje que poseen estudiantes de pedagogía de la Universidad de Magallanes (Punta Arenas, Chile) y docentes en ejercicio en la educación básica de la ciudad de Punta Arenas, Chile. La muestra no probabilística compuesta por 121 participantes en total, respondieron un cuestionario de dilemas. Estos ofrecen alternativas de resolución, representando concepciones de aprendizaje, directa, interpretativa, constructivista y posmoderna. Se compararon las concepciones de aprendizaje de estudiantes nuevos, estudiantes avanzados, docentes con poca experiencia y docentes con mayor experiencia. Los resultados mostraron que las diferentes concepciones están presentes en los sujetos a la hora de resolver dilemas de la práctica docente. Predominan las concepciones constructivistas en estudiantes de último año y docentes en ejercicio, quienes difieren significativamente de los estudiantes de primer año. Se discute el efecto que podría tener la formación docente universitaria y continua; en estos resultados, y en general en la construcción del saber pedagógico de estudiantes y profesores.
\end{abstract}

Palabras clave: concepciones de aprendizaje, formación docente, constructivismo, concepciones del profesorado, concepciones de estudiantes de pedagogía

\section{Learning Conceptions by Students of the Elementary School Teacher's Program of the University of Magallanes and Elementary Schools Teachers working in the City of Punta Arenas, Chile}

\begin{abstract}
The aim of the present study was to know and analyze the learning conceptions by students of the elementary school teacher's program at University of Magallanes in Chile and teachers already working at elementary schools in the city of Punta Arenas. The non-random sample consisted of 121 participants, who answered a questionnaire with dilemmas. The dilemmas could be solved by choosing a direct, interpretative, constructivist, or postmodern learning conception. Learning conceptions were compared between young students, advanced students, recently graduated teachers and experimented teachers. Results show that all of the different conceptions were present when the subjects solved the problems from pedagogical praxis but that there was a predominance of the constructivist approach in senior students and teachers, who differed significantly from the freshmen students. The possible effects of higher education and teacher training programs on the current results and on the pedagogical knowledge of students and teachers are discussed.
\end{abstract}

Keywords: learning conceptions, teacher training, constructivism, teachers' conceptions, teacher trainees conceptions 


\section{INTRODUCCIÓN}

En la década de los noventa, en Chile se inició un proceso de reforma educacional, que se orientó al logro de una educación de calidad distribuida con equidad (García-Huidobro y Cox, 1999), para lo cual proponía una serie de cambios basados en políticas educativas (distribución de recursos, extensión horaria, jornada escolar completa, entre otros) y en estrategias metodológicas, para que los docentes las implementaran (cambios en las prácticas pedagógicas, sistemas de evaluación, entre otros). En este contexto, la apuesta más relevante, fue la sustitución de paradigma, desde uno realista a uno constructivista, y se esperaba que en la medida que los docentes se capacitaran y "sintonizaran" con los cambios de paradigma, con base epistemológica constructivista (Gómez y Guerra, 2012; Rosas y Sebastián, 2008), cambiarían sus prácticas, incidiendo positivamente en la calidad de la enseñanza y la educación. Veintidós años después, los estudios muestran resultados menores que los esperados en relación a la inversión, y una percepción en los propios docentes de una reforma difícil de implementar o (im)practicable (véase, por ejemplo, Paukner-Nogués, SanhuezaHenríquez y San Martín-Ramírez, 2012).

Ante esto es posible plantear múltiples explicaciones, sin embargo, adquiere gran relevancia la idea de que ante las demandas de este cambio, podríamos estar observando intentos por modificar las prácticas, pero disociado de las creencias. Pozo et al. (2006) afirman que si se quieren cambiar las prácticas, las formas de hacer, se deberían necesariamente cambiar las concepciones implícitas que les subyacen o al menos estar conscientes de tales creencias y de su uso. Posición que en todo caso es discutible, dado que la relación entre creencias y acción es compleja, y existe evidencia respecto a la posibilidad de cambio en las prácticas sin necesariamente haberse producido cambios radicales a nivel de teorías personales (Castro, 2012). Por otra parte, existen antecedentes teóricos y empíricos respecto a la dificultad de cambio de las creencias, tanto en el profesorado en ejercicio como en los estudiantes de pedagogía ( $\mathrm{Ng}$, Nicholas y Williams, 2010). Adicionalmente existe evidencia en el estudio de las concepciones del profesorado, del sustento de creencias que favorecen la mantención de las acciones de los docentes por sobre el cambio de ellas (Bortoluzzi y Catalán, 2014), situación que podría explicar el no cambio en sus prácticas pedagógicas.

Acorde a lo anterior, un supuesto del denominado paradigma del "pensamiento del profesor" es que el comportamiento de los docentes se guía por pensamientos, juicios y decisiones (Martín et al., 2011; Shavelson y Stern, 1989), es decir, por sus concepciones o teorías personales. Así, desde este enfoque importa conocer las concepciones de los docentes por la relevancia que tendrían al influir sus prácticas pedagógicas. De acuerdo a Catalán (2011) estas teorías subjetivas o implícitas son parte del conocimiento profesional docente, estas podrían ser definidas entonces como teorías pedagógicas personales construidas sobre la base de conocimientos pedagógicos históricamente elaborados y transmitidos a través de la formación y en la práctica pedagógica (Rodrigo, Rodríguez y Marrero 1993: 245).

Es necesario tener en cuenta que estos términos surgen desde una pregunta previa, relacionada a cómo es que el docente se representa y acciona uno de los conceptos centrales de su quehacer, nos referimos al aprendizaje, cómo es que conceptualiza aprender y cómo es que aprende a conocer el modo en que se estimula y se desarrolla en los alumnos. Estas preguntas son formuladas desde la inquietud de pensar en el docente como quien se sumerge en una profesión que tiene una característica particular, y es que probablemente es una de las pocas profesiones en que se llega a aprender a enseñar, con la particularidad que en la propia historia personal el docente fue el sujeto a quien se le enseñó. La experiencia previa de haber sido alumno y haber sido el mismo sujeto al cual se quiere conocer debe ahora enfrentarse a la teoría y encontrar su ajuste, si se desea, o disponerse a un conflicto, "conflicto cognitivo" (Schnotz, Vosniadou y Carretero, 2006) o una tensión entre sus propósitos personales y profesionales (Cerpa, 2009: 205). Este conflicto a veces verbalizado en frases como: "yo aprendí así... ¿por qué ahora habría que pensar que ahora aprenden de otro modo?...", muestra que hay una experiencia, real, personal y significativa que sale a una discusión con otro modo de explicar un fenómeno conocido, pero ahora definido o entendido como conocimiento científico. Respecto a las concepciones sobre la enseñanza y aprendizaje que adquieren los docentes durante su formación inicial o permanente, estudios muestran que estos significados y sentidos con los que enfrentan su proceso de formación han sido construidos desde la infancia en el seno de sus familias (véase, por ejemplo, Castro, 2012) en el medio social y en el escolar, con la dificultad de que a veces no son advertidas por los procesos de formación (Oliver Vera, 2009), por lo que persisten sin mayores cambios e influyen el desempeño del docente.

\section{Formación y perfeccionamiento profesional docente en Chile}

"Las tendencias educativas del nuevo siglo han impuesto una dinámica diferente y más rigurosa en relación con el modo de actuación profesional de los docentes" (Acosta et al., 2015). En ese contexto de demandas de cambio y mejora, la formación de docentes en Chile se relaciona con los niveles o modalidades del sistema 
escolar, dando origen a distintas carreras pedagógicas para formar docentes en educación Preescolar, Básica (Primaria), Diferencial y Media (Secundaria). No obstante, la autonomía universitaria da lugar a que cada institución diseñe sus propios programas. En los últimos años, se han realizado esfuerzos de coordinación entre el Ministerio de educación y las universidades para analizar el currículo escolar, mediante seminarios y jornadas de estudio, pero de carácter limitado en cuanto a cobertura y extensión. La OCDE, en su revisión de políticas educacionales de Chile del año 2004, señalaba la poca relación entre los currículos de formación inicial en las universidades y el currículo escolar, hoy en día el diagnóstico sigue mostrando este débil vínculo entre el campo disciplinar de la educación y el sistema escolar, en la formación docente (Cox, 2012). Un informe OCDE (2004) señalaba que el 96\% de las carreras presentaba "un grado de descoordinación entre las unidades que imparten la carrera, lo que ha tenido un impacto negativo en la coherencia e integridad de la formación que se entrega" (Ministerio de Educación, 2005:40). El mismo informe, señalaba la importancia de propiciar una carrera académica que incentivara la innovación.

El perfeccionamiento y la actualización del docente son un posible vehículo de cambio en las concepciones y teorías de los docentes, y en Chile es también un factor de aumento de la remuneración. Toda la oferta de formación continua para docentes, está impulsada en su mayoría por el Ministerio de Educación, a través de su organismo especializado, el Centro de Perfeccionamiento, Experimentación e Investigaciones Pedagógicas (CPEIP). En ella se contemplan diversas acciones, destacándose, entre otras, cursos de actualización disciplinaria y pedagógica, postítulos de especialización, etc. estas ofertas son de bajo costo o gratuitas, de participación voluntaria y de acceso limitado. Se estima que un número superior a 25.000 docentes participan anualmente en dichos programas, cifra que fue mayor en el período de introducción del nuevo currículo de Educación Básica y Media (1997 - 2002).

El Ministerio de Educación de Chile en el año 2000, publicó los estándares para la formación inicial de docentes, que resultó de un trabajo conjunto de académicos de instituciones formadoras, docentes de aula, colegio de docentes y Ministerio de Educación, con el fin de establecer parámetros que indiquen qué es lo que el profesor debe saber y poder hacer a su egreso, para que sirvieran de orientadores de los contenidos curriculares y las estrategias de formación de los estudiantes de pedagogía. Según el informe de la comisión sobre formación inicial docente, se señala que varias instituciones utilizaron los estándares como referentes en la formación de pedagogos, pero sólo una de ellas los utilizaba como criterios de evaluación del desempeño de los estudiantes a su egreso. Es decir, una política de formación inicial docente caracterizada por prescribir, pero "con consecuencias bajas" cuando los resultados no son los esperados, situación que habría cambiado en los últimos años con la introducción de exámenes con incentivos, convenios de desempeño y becas (Cox, 2012). Recientes evidencias internacionales, muestran cómo la inclusión de una cultura de evaluación en el profesorado, desde su formación inicial, impacta en sus concepciones acerca de, por ejemplo, su propia eficacia como docentes (véase, por ejemplo, van Dinther, Dochy y Segers, 2015).

\section{Concepciones o teorías implícitas sobre el aprendizaje}

Las concepciones de los docentes han sido estudiadas desde el enfoque de sus teorías implícitas (o subjetivas) postulando que tendrían un papel importante en la forma en que éstos representan lo educativo, lo que ha sido denominado por Janssens y Kelchtermans (1997) como teacher's subjective educational theory, encontrándose actualmente investigaciones situadas en un nivel de indagación general que dan cuenta de su implicancia en el ejercicio profesional (Catalán, 2010), su propia formación (Cuadra y Catalán, en prensa), los procesos de reflexión (Castro et al., 2012) y de cambio mediante la formación continua (Castro y Cárcamo, 2012) e inicial (Seebauer, 2009), la enseñanza y el aprendizaje (Cuadra, 2009), y estudios que abordan temáticas o ámbitos educativos específicos tales como la violencia en las escuelas (Von Reininghaus, Castro y Frisancho, 2013), la motivación y las expectativas que tienen del éxito y fracaso escolar (Castro et al., 2012), la participación en la toma de decisiones escolares (Gastager et al., 2010), las explicaciones acerca de algún contenido de enseñanza, la comprensión del lenguaje (Makuc, 2011), actuando como una especie de filtro que organiza sus prácticas pedagógicas (Gómez-López, 2008) y predispone los procesos de cambio (Limón, 2002).

Algunos estudios de teorías implícitas de docentes, han dado cuenta como parte de sus hallazgos, de la simplicidad de este tipo de representaciones (Cuadra, 2009; Jiménez y Feliciano, 2006), esto es, explicaciones sencillas de fenómenos educativos complejos y de una tendencia a la escasez de las mismas o del nivel preteórico que presentan (Catalán, 2004), situación que podría asociarse a limitaciones en su quehacer profesional y a la necesidad de promover un pensamiento de mayor elaboración. También se han presentado evidencias del bajo control que los docentes consideran poseen sobre los factores que influyen en los procesos de enseñanza y aprendizaje del estudiante. Implica principalmente una atribución externa del 
control, lo que estaría ligado con una menor agentividad (Castro y Cárcamo, 2012; Castro et al., 2012). Lo anterior, da cuenta de la necesidad de que la formación del profesor considere la subjetividad del mismo, dado el impacto que ésta tiene en sus prácticas pedagógicas (Cuadra y Catalán, en prensa; European Educational Research Association, 2008).

Sería posible afirmar que estas concepciones o teorías implícitas acerca del aprendizaje que sustentan los docentes pueden cambiar, esto en función del nivel de pericia o bien, como parte de un mecanismo evolutivo de desarrollo (Pozo y Scheuer, 1999). Lo anterior es coherente con quienes postulan que el docente se va formando como un profesional de la educación a través de una serie de experiencias formales e informales de aprendizaje, ocurridas durante toda la vida (Cuadra y Catalán, en prensa), las que influirían en la concepción de aprendizaje a la que adhiera. Diversas investigaciones han indagado en estas concepciones del aprendizaje (véase, por ejemplo, Martínez, 2004), utilizando diversas tipologías para definir las concepciones de aprendizaje, en este estudio en particular nos basamos en la categorización que realizan Pozo y Scheuer (1999), quienes definen cuatro concepciones de aprendizaje: i) teoría directa, la cual parte del supuesto de que el conocimiento es una copia fiel de la realidad, reduce el aprendizaje a un hecho, es reproducir el mundo, desde el punto de vista epistemológico, se hablaría de realismo ingenuo (Martín et al., 2011:171); ii) teoría interpretativa, plantea que la actividad personal del aprendiz es imprescindible para lograr un buen aprendizaje, mantiene la idea de que la ejercitación reiterada es necesaria para apropiarse del conocimiento y que la observación activa involucra la intervención de algunos procesos psicológicos; iii) teoría constructiva, asume que el aprendizaje implica procesos de reconstrucción que generan nuevas relaciones y conocimientos, implica una reelaboración del objeto aprendido, esta concepción niega el saber absoluto y se apropia del relativismo en todo conocimiento; iv) teoría posmoderna, la cual asume un relativismo radical, tiene una base epistemológica similar al constructivismo, pero señala que todo conocimiento es situado, y por lo tanto tiene validez y se construye sólo en ese contexto (Pozo et al., 2006).

A partir de lo anterior, la presente investigación se propone describir las concepciones de aprendizaje que tienen distintos grupos de individuos relacionados a la educación: alumnos de primer año en la carrera de pre-grado de pedagogía básica, alumnos de último año de la misma carrera y pedagogos de enseñanza básica que tengan hasta dos años de experiencia docente y más de quince años de práctica docente. Con esta comparación se busca iniciar un análisis que permita orientar la respuesta al problema de identificar cómo es que los docentes forman sus concepciones acerca de los procesos de aprendizajes de sus alumnos, si de un modo natural e informal, producto de la formación universitaria o de la práctica pedagógica. Además de identificar la compatibilidad de estas concepciones de aprendizaje con las propuestas y presupuestos constructivistas de la última reforma curricular chilena (Gómez y Guerra, 2012; Rosas y Sebastián, 2008; Donoso, 2005).

Así surgen las siguientes preguntas de investigación: ¿cuáles son las concepciones de aprendizaje que tienen los estudiantes de pedagogía y los docentes en ejercicio?, ¿Existen diferencias entre los diferentes grupos de estudiantes y docentes en la identificación con alguna de las teorías o concepciones de aprendizaje?, y ¿qué grupos difieren entre sí? Hipotetizamos que los estudiantes nuevos tendrán mayor identificación con la teoría directa, debido a su experiencia escolar y menor identificación con las teorías interpretativas y constructivistas del aprendizaje que los demás grupos, ya que hasta ahora no han recibido una instrucción formal sobre teorías del aprendizaje. Por su parte creemos que los estudiantes de último año y docentes en ejercicio, han recibido entrenamiento formal en teorías constructivistas del aprendizaje, por lo que esperamos que sea la teoría que predomine en esos grupos.

\section{MÉTODO}

Participantes. La muestra no probabilística intencionada, estuvo compuesta por 121 participantes distribuidos en cuatro grupos diferentes, dos de ellos por estudiantes de Pedagogía Básica de primer año $(n=31)$, y cuarto año de formación $(n=21)$, y dos grupos de docentes en Ejercicio, uno con no más de 2 años de ejercicio profesional ( $n=33$ ), y otro grupo de docentes con más de 15 años de ejercicio profesional $(n=36)$. En la muestra total, la mayoría de los participantes fueron mujeres (79.3\%) y dentro de cada subgrupo también la muestra femenina está sobrerrepresentada (>80\%) excepto en el grupo de docentes nuevos donde los varones representan el $39.4 \%$, no obstante, la variable género no presenta diferencias significativas en ninguna de las cuatro concepciones del aprendizaje. 
Instrumento: Se utilizó el Cuestionario de Concepciones del Profesorado de Primaria (Martín et al., 2001) que consiste en una serie de 18 dilemas (ver Ejemplo) que representan una situación particular que ocurre en la escuela y donde cada dilema ofrece cuatro posibles formas de resolverlo desde el punto de vista de un docente en ejercicio, cada una representando a una concepción del aprendizaje distinto (directa, interpretativa, constructiva y posmoderna), además los dilemas representan tres diferentes escenarios: Capacidades y Contenidos (6), Motivación (5), y Evaluación (7). Los dilemas son contestados en una planilla con las cuatro posibles alternativas para cada situación, en la que el entrevistado sólo puede elegir una respuesta. El total de dilemas entrega un resultado de frecuencias de respuestas en cada una de las concepciones de aprendizaje representadas. Los dilemas que hacen referencia al escenario de capacidades y contenidos pretenden explorar cómo los docentes eligen los contenidos y cómo enseñarlos. Por otra parte, los referidos a la motivación, buscan indagar qué tipo de teorías o concepciones los docentes atribuyen al papel de la motivación en el aprendizaje y finalmente los dilemas relacionados a los procesos de evaluación permiten conocer las concepciones de aprendizaje en la tarea específica de tener que evaluarlos en sus alumnos (Martín et al., 2006). En otros estudios, este instrumento ha reportado un índice de confiabilidad Alfa de Cronbach de .74 en poblaciones de docentes en ejercicio y estudiantes de pedagogía de Chile (Gómez y Guerra, 2012), no obstante, en nuestro estudio este índice resultó bajo (Cronbach's $=.40$ ). Sin embargo, dado que no se espera que las diferentes dimensiones correlacionen entre sí, ya que un individuo puede tener diferentes teorías presentes dependiendo el tipo de dilema que debe enfrentar, no resulta esperable para este instrumento un alto índice.

Tabla 1: Ejemplo de Dilema del Cuestionario de Concepciones del Profesorado de Primaria (Martín et al., 2001

\footnotetext{
Dilema:

Es cada vez más frecuente que los profesores de Básica se preocupen porque los alumnos muestran cada vez menos interés por aprender. Entre las razones que los profesores dan para explicar esa falta de interés y las medidas que proponen para intentar resolver el problema se encuentran las siguientes:

a) El problema se debe a que los contenidos escolares están muy alejados de los intereses de los alumnos. La mejor manera de despertar el interés de los alumnos es crear un clima relajado en clase para que se sientan a gusto y hacer más atractivos y amenos los contenidos.

b) Ciertamente, los contenidos escolares se alejan de los intereses de los alumnos. Lo que habría que hacer es seleccionar los contenidos que más les puedan interesar y así no les resultarán tan costosos.

c) El problema se debe a que cada vez se les exige menos en su vida y por eso están poco acostumbrados a esforzarse. Lo que hay que hacer es fomentar la cultura del esfuerzo, exigiéndoles más y recompensándoles de acuerdo con sus logros.

d) La falta de interés por aprender que muestran los alumnos se debe a que no llegan a comprender los contenidos que les enseñamos. Habría que procurar partir de los significados que ellos dan a las cosas para irse aproximando a los significados que queremos que aprendan.
}

Para el ejemplo de dilema descrito, cada individuo que responde el cuestionario debe inclinarse por sólo una opción de las cuatro posibles formas de responder ante tal situación. Este dilema hace referencia a un escenario de motivación, y la alternativa a) sería una respuesta que refleja una teoría interpretativa; la alternativa b) representa una explicación basada en la teoría posmoderna; la alternativa c) sería parte de una teoría directa; y por último la alternativa d) representaría una respuesta de la teoría constructivista.

\section{Procedimiento}

Se invitó a participar a estudiantes de la carrera de Pedagogía de Educación Básica de primer y cuarto año de la Universidad de Magallanes para contestar el Cuestionario de Dilemas de manera simultánea en cada grupo, por su parte los docentes en ejercicio fueron contactados en establecimientos educacionales municipales de educación básica e invitados a participar, quienes respondieron el cuestionario de manera individual. Todos los estudiantes de Pedagogía accedieron a responderlo en una sesión de una hora.

\section{RESULTADOS}

En relación a la primera pregunta de investigación, notamos que a excepción del grupo formado por estudiantes de primer año, los demás grupos presentaron una distribución bastante similar en las preferencias de sus respuestas, adhiriendo mayoritariamente a las concepciones constructivas del aprendizaje $(M=38.40$, $D S=13.37)$, luego a las teorías interpretativas $(M=24.94, D S=10.41)$, quedando las concepciones directa 
$(M=18.15, D S=10.12)$ y posmoderna $(M=18.51, D S=9.82)$ como las que menos identifican su ideas acerca de cómo actuar en una acción pedagógica. La Tabla 2 muestra que en el grupo de estudiantes nuevos, las respuestas se distribuyeron entre las distintas teorías, sin observarse una preferencia determinada.

Tabla 2. Distribución de promedios en las respuestas de cada grupo para las distintas concepciones de aprendizaje. En la Tabla se presenta promedio y desviación estándar en paréntesis para cada concepción de aprendizaje.

\begin{tabular}{lcccc}
\hline & \multicolumn{4}{c}{ Teorías } \\
\cline { 2 - 5 } & Directa & Interpretativa & Constructivista & Posmoderna \\
\hline Estudiantes Primer Año & $18.82(8.32)$ & $29.92(11.80)$ & $25.27(11.37)$ & $25.99(9.44)$ \\
Estudiantes Cuarto Año & $19.84(11.73)$ & $26.19(10.11)$ & $37.30(10.41)$ & $16.67(9.78)$ \\
docentes $<2$ años experiencia & $18.52(8.63)$ & $22.73(10.97)$ & $40.07(16.12)$ & $18.69(10.74)$ \\
docentes $>15$ años experiencia & $16.82(10.50)$ & $26.23(9.99)$ & $37.50(12.27)$ & $19.44(9.06)$ \\
\hline
\end{tabular}

En un segundo análisis nos propusimos identificar diferencias significativas entre los grupos por cada una de las teorías o concepciones del aprendizaje utilizando la prueba One-Way ANOVA (Análisis de la Varianza de una vía), así pudimos establecer que en la muestra sólo se observan diferencias significativas entre los grupos en las teorías constructivista $\left(F_{(3,117)}=8.18, p=.001\right)$ y posmoderna $\left(F_{(3,117)}=4.86, p=.003\right)$, mientras que en las concepciones directa e interpretativa, no existen diferencias significativas en los promedios obtenidos por los diferentes grupos que componen la muestra. Comparaciones Post hoc usando la prueba Tukey HSD indicaron que en la concepción constructivista, el promedio de respuestas del grupo de estudiantes de primer gño es el que significativamente puntúa más bajo que los otros tres grupos, con un efecto grande de la medida $(2=0.17)$, sin existir diferencias significativas entre los demás grupos (ver figura 1).

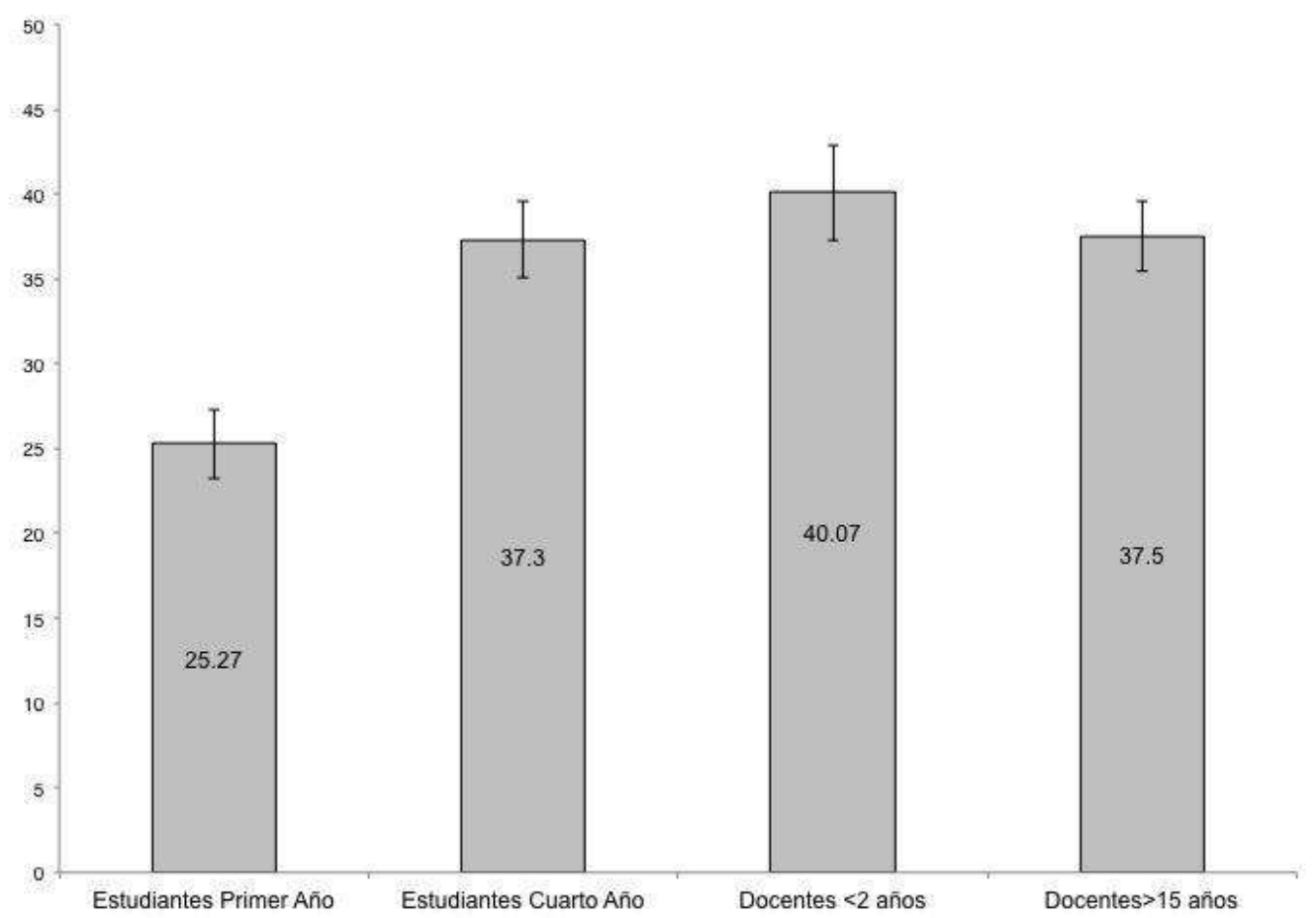

Fig. 1: Promedios de respuestas constructivistas en los dilemas por cada grupo y tamaños de las diferencias significativas.

En oposición a lo anterior, utilizando el mismo tipo de análisis en la concepción posmoderna, el grupo de estudiantes de primer año se diferenció significativamente de todos los demás grupos con un efecto mediano de la medida $\left(l^{2}=0.11\right)$, obteniendo un promedio mayor, sin existir diferencias entre los demás grupos (ver figura 2). Debido a la diferencia leve entre los tamaños de la muestra, analizamos las diferencias para ambas concepciones, usando la prueba Post hoc Gabriel, con la que se obtuvieron los mismos resultados descritos 
anteriormente. No se presentaron otras diferencias en la muestra, y en la teoría directa e interpretativa no hubo ningún tipo de diferencia significativa en los promedios de respuestas entre los grupos.

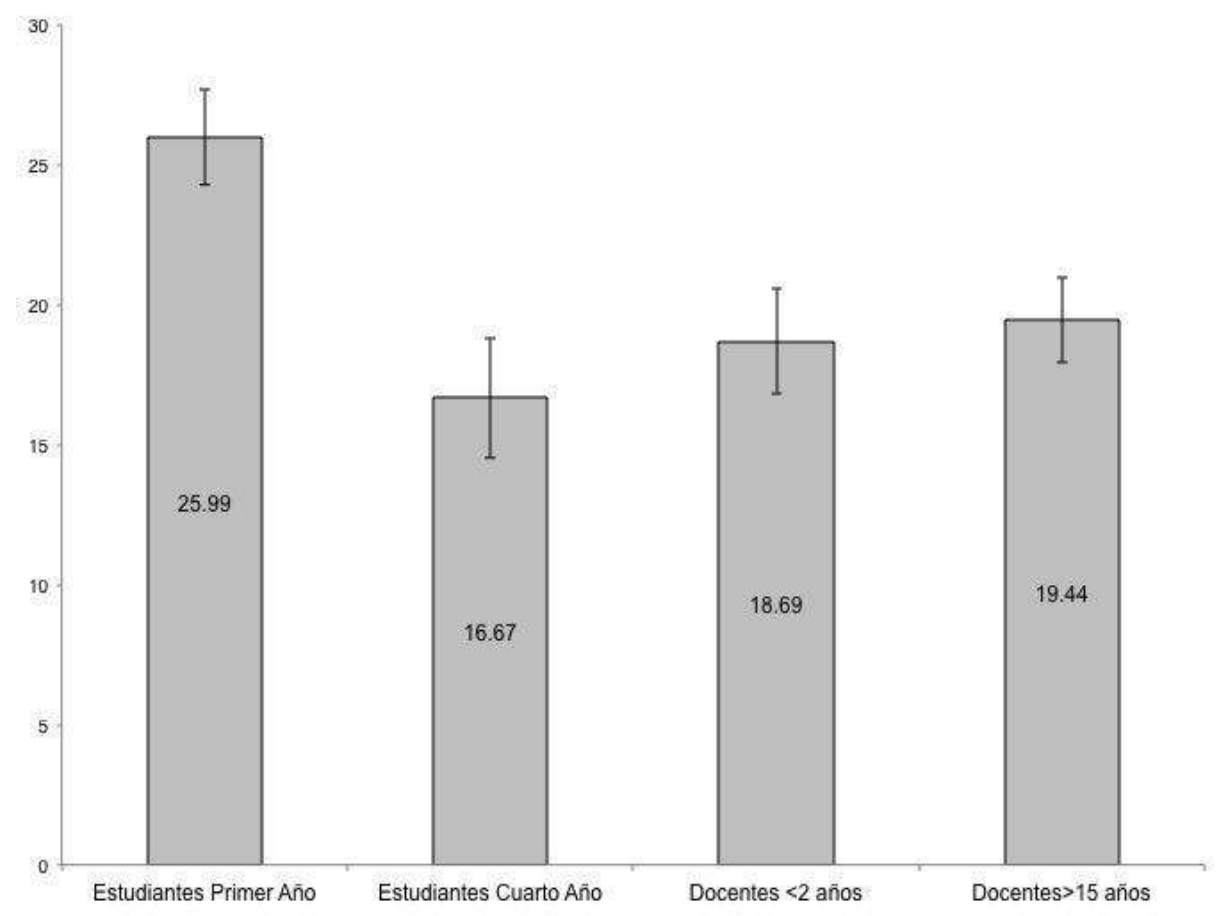

Fig. 2: Promedios de respuestas Posmodernas en los dilemas por cada grupo y tamaños de las diferencias significativas.

\section{DISCUSIÓN}

Este estudio nos permite indagar en un aspecto relevante para la formación docente, ya que intenta aportar información acerca de si las concepciones que los estudiantes y docentes de pedagogía tienen acerca del aprendizaje, tienen por un lado consistencia entre sus actores y por otro lado, si estas concepciones son coherentes a las teorías que la formación formal y los enfoques actuales en educación pretenden instaurar como parte de un saber pedagógico. En primer lugar, se observa en la muestra, que ninguno de los grupos presenta una teoría exclusiva que refiera sus concepciones o teorías implícitas sobre el aprendizaje, sobre todo los estudiantes de primer año, quienes responden a los dilemas utilizando casi en la misma proporción las cuatro concepciones del aprendizaje. Por otro lado, los demás grupos, estudiantes de último año y docentes en ejercicio profesional, tampoco muestran un uso exclusivo de alguna de las teorías para resolver los dilemas, pero sí tienen una mayor inclinación a resolver los dilemas con una perspectiva constructivista, incluyendo en ciertas situaciones, perspectivas también directas, interpretativas y en menor medida posmodernas a la resolución de estos dilemas, lo que refuerza nuevamente una de las conclusiones de los autores del instrumento en estudios previos, que los docentes parecen ser "constructivos, pero no tanto" (Martín et al., 2011:179) y también en estudios en Chile (Gómez y Guerra, 2012; Judikis, Estrada, Makuc, Molina y Cárcamo, 2008).

La no presencia de teorías exclusivas en los docentes, podría estar indicando flexibilidad en las concepciones de los docentes en relación al contexto o demanda. Es decir, ante cambiantes escenarios cotidianos de la práctica pedagógica, el profesor activa diferentes teorías que le permiten explicar, justificar o predecir sus acciones. Lo anterior parece una situación deseable, dicho de otra forma, no parece razonable postular ni propiciar la adherencia de los docentes a una única teoría acerca de la enseñanza y el aprendizaje (ni siquiera la constructivista), dado que el contexto escolar es diverso y en particular la naturaleza de los diferentes tipos de aprendizaje, exige la "coexistencia" de concepciones alternativas (Castro, 2012; Rodrigo y Correa, 2001). Además, como señalan Estévez-Nenninger, et al. (2014), las creencias del profesorado que se orientan por nuevos paradigmas, como en este caso podría tratarse del enfoque constructivista, conviven con creencias tradicionales, sin negar la generación de cambios paradigmáticos posibles de producirse en procesos de capacitación y experiencia docente.

Es interesante también discutir respecto a cómo los presentes resultados también abordan la cuestión que Gómez y Guerra se preguntaban en un estudio similar en Chile si: ¿se mantiene la tendencia mostrada por los estudios de los años 90 que señalan la falta de impacto de los programas de formación, en la 
transformación de la visión realista con la que ingresan a la universidad? (Gómez y Guerra, 2012:28). Al respecto, estos hallazgos muestran un cambio en esta tendencia, al sugerir que tanto en los estudiantes de pedagogía de último año, como en los docentes en ejercicio profesional, la teoría directa vinculada a la visión realista, carece de predominancia y es en menor medida, el modo en que los participantes del estudio resolvieron los dilemas. No obstante, debemos considerar que un diseño como el de este trabajo no permite afirmar si este cambio sería o no producto de la formación o de otras fuentes de influencia, sin embargo, es posible hipotetizar, a partir de los resultados, que la formación universitaria y la formación continua han generado un impacto en las creencias y concepciones que los actores de la educación tienen acerca de cómo los niños aprenden y cómo resolver problemas cotidianos desde el punto de vista pedagógico, ya que están en mayor medida presentes las respuestas de tipo constructivista, que precisamente representan el paradigma que se ha intentado promover en los espacios formales de capacitación pedagógica post reforma educacional.

Aquí llama la atención que, incluso en aquellos docentes con más experiencia y que fueron formados en tiempos donde el paradigma predominante en educación era más bien aquellos relacionados a las teorías directas, han internalizado, al menos al nivel de teorías implícitas, ideas y formas de resolver acciones pedagógicas desde un paradigma diferente. Esto permite ver con cierto optimismo la capacidad del sistema de formación universitaria y de los variados programas de capacitación continua, en su capacidad de generar cambios en las concepciones y las teorías implícitas con las que enfrentan al menos conceptualmente, desafíos pedagógicos cotidianos. Decimos con cierto optimismo, dado que queremos enfatizar que la posición asumida aquí por los autores de este trabajo es la del desarrollo de un profesional docente con capacidad de identificar diversas posiciones teóricas personales o epistemológicas que le permitan operar con mayor efectividad ante los también diversos escenarios que le demanda la práctica cotidiana. No existe una sola teoría del aprendizaje que logre explicar las diferentes naturalezas de este (Pozo, 2008) y por tanto no sería la situación deseable que los docentes adhieran rígidamente a una sola concepción.

En relación a lo anterior, el estudio refleja algunas de sus limitaciones como, por ejemplo, la recolección de datos realizada en una sola medición, por lo que no podemos asegurar que las concepciones de los sujetos participantes de la muestra realmente han sufrido modificaciones a lo largo de su trayectoria, ya sea académica o profesional. Para esto sería necesario realizar un estudio longitudinal, con medidas pre y postest, que puedan medir el impacto de la formación y de la experiencia profesional o formación continua en el cambio de las concepciones sobre la enseñanza y el aprendizaje, lo cual podría también establecer la consistencia de este instrumento en medidas en distintos tiempos.

Por otra parte, este estudio indaga en las concepciones o teorías implícitas, pero no en las prácticas pedagógicas concretas que estos docentes despliegan en el aula, por lo que no podemos afirmar, por ejemplo, que este predominio de concepciones constructivistas necesariamente se relaciona a una acción pedagógica por parte de esos docentes de tipo constructivista, aunque sí hemos obtenido algunas evidencias previas (véase, por ejemplo, Judikis et al., 2008) que señalan un alto grado de concordancia entre las teorías implícitas que tienen los docentes acerca del aprendizaje y sus prácticas en el aula, medidas a través de observación directa.

Finalmente podemos señalar que diversos estudios, tanto en Chile como en otros países, han arrojado evidencia similar (Gómez y Guerra, 2012; Judikis et al., 2008; Martín et al., 2011; Martín et al., 2006; Pérez Echeverría et al., 2006), mostrando un predominio de concepciones constructivistas en docentes de diferentes niveles de formación y años de experiencia en aula, por medio de diversos métodos para evaluar estas concepciones, lo cual otorga visibilidad del impacto que tendría la formación universitaria y continua en el saber pedagógico especializado.

\section{CONCLUSIONES}

De los resultados obtenidos, de su análisis y de su discusión, se pueden obtener las siguientes conclusiones, sobre la aplicación a esta muestra del "Cuestionario de Concepciones del Profesorado de Primaria":

1.- La mayoría de los grupos estudiados adhieren a las concepciones constructivistas del aprendizaje.

2.- En el grupo de estudiantes nuevos no se observa una preferencia alguna de las concepciones de aprendizaje.

3.- Sólo se observan diferencias significativas en los grupos en las teorías posmoderna y constructivista. 
4.- Los presentes resultados son coherentes con otros estudios que muestran un predominio de la concepción constructivista en docentes, cuando las concepciones son medidas a través de cuestionarios con características similares al utilizado en este estudio.

Para finalizar creemos que es necesario seguir complementando estas formas de evaluar las concepciones o teorías docentes acerca del aprendizaje mediante dilemas, con métodos cualitativos de indagación que nos permiten comprender la relación entre las concepciones de los docentes y sus prácticas pedagógicas, desde las propias explicaciones de los docentes.

\section{REFERENCIAS}

Acosta, L. A; O. Abreu y M.F, Coronel, Sistema de Formación Pedagógica en la Universidad de Otavalo en Ecuador, http://dx.doi.org/10.4067/S0718-50062015000200007, Form. Univ., La Serena, (8)2, (2015)

Bortoluzzi, M. y J. Catalán, Teorías subjetivas de profesores en reuniones de trabajo: un estudio descriptivointerpretativo, doi: 10.1590/S1413-85572014000100016, Revista Quadrimestral da Associação Brasileira de Psicologia Escolar e Educacional, SP. 18(1), 151-159 (2014)

Castro-Carrasco, P.J., Cambio de teorías subjetivas de profesores: respecto a la enseñanza y el aprendizaje de valores. EAE, Saarbrücken, Alemania (2012)

Castro, P., C. Agüero, A. Barraza, G. Escobar y J. Jorquera, Disposición a la Reflexión Colectiva Sistemática en Docentes de un Centro Educativo de Chile, http://www.scielo.org.mx/scielo.php?pid=S140566662012000200012\&script=sci_arttext, ISSN 1405-6666, Revista mexicana de investigación educativa, 17(53), 573-591 (2012)

Castro, P. y R. Cárcamo, Cambio de Teorías Subjetivas de Profesores Respecto a la Educación en Valores, http://www.scielo.org.ar/scielo.php?script=sci_arttext\&pid=S1852-73102012000100001, ISSN 1852-7310, Subjetividad y procesos cognitivos, 16(1) 17-42 (2012)

Catalán, J., Formación Inicial de Educadoras de Párvulos: un Estudio de Caso desde las Teorías Subjetivas de Formadores y Formadoras, http://www.rieoei.org/deloslectores/738Catalan.PDF, ISSN: 1681-5653, Revista Iberoamericana de educación, (33)5 1-14 (2004)

Catalán, J., Del pensamiento al conocimiento profesional del profesor. En: Psicología Educacional: Proponiendo rumbos, problemáticas y aportaciones, J. Catalán editor. Editorial Universidad de La Serena, La Serena, Chile (2011)

Cerpa, C.D., Teorías subjetivas de profesores acerca del aprendizaje escolar. Tesis para optar al grado de Magíster en Psicología, Departamento de Psicología de la Universidad de La Serena, La Serena-Chile (2009)

Cox, C., Formación de Docentes en la Encrucijada. Dictada en la Universidad de La Serena, La Serena, Chile, 29 de Marzo de 2012 (2012)

Cuadra, D. y J. Catalán, Teorías Subjetivas en Profesores acerca de su Formación Profesional, ISSN 14132478, Revista Brasileira de Educação (en prensa)

Cuadra, D., Teorías Subjetivas en Docentes de una Escuela de Bajo Rendimiento, sobre la Enseñanza y el Aprendizaje del Alumno, http://www.scielo.org.mx/scielo.php?pid=S140566662009000300015\&script=sci_arttext, ISSN 1405-6666, Revista Mexicana de Investigación Educativa, 14(42), 939-967 (2009)

Donoso, S.E., Reforma y Política Educacional en Chile 1990-2004: El Neoliberalismo en Crisis. Estudios Pedagógicos, 31(1), 113-135 (2005)

Estévez-Nenninger, E.H., Á.A. Valdés-Cuervo, C.G. Arreola-Olivarria y M.G. Zavala-Escalante, Creencias sobre Enseñanza y Aprendizaje en Docentes Universitarios, doi:10.11144/Javeriana.M6-13.CSEA, Revista Internacional de Investigación en Educación, 6(13), 49-64 (2014) 
European Educational Research Association, Individualised Teaching and Learning - The "Indive Project" (en linea), 2008. http://www.indive.net/resources/Ecer_Abstract.pdf. Acceso: 17 de abril (2015)

García-Huidobro, J.E. y C. Cox, La reforma educacional chilena 1990-1998. Visión de conjunto. En: La Reforma educacional chilena, J. García-Huidobro editor. Editorial Popular, Madrid, España (1999)

Gastager, A., J. Patry y A. Wiedemair, Subjective Theories about Participation at School, Presentado en el $2^{\circ}$ Symposium of EARLI SIG 13, Tel Aviv-Israel, 30 de Agosto al 2 de Septiembre de 2010, http://www.unisalzburg.at/fileadmin/oracle_file_imports/1817280.PDF. Acceso: 17 de abril (2015)

Gómez-López, L., Las teorías implícitas de los profesores y sus acciones en el aula, http://rei.iteso.mx/handle/11117/1357, Sinéctica, 30, 1-22 (2008)

Gómez, V.D. y P. Guerra, Teorías implícitas respecto a la enseñanza y el aprendizaje: ¿Existen diferencias entre profesores en ejercicio y estudiantes de pedagogía? Estudios Pedagógicos, 38(1), 25-43 (2012)

Janssens, S. y G. Kelchtermans, Subjective Theories and Professional Self of Beginning Teachers, http://files.eric.ed.gov/fulltext/ED408252.pdf, Paper presented at the annual meeting of the American Educational Research Association. Chicago, USA, 1997. Acceso: 17 de abril (2015)

Jiménez, A. y L. Feliciano, Pensar el pensamiento del profesorado, http://revistadepedagogia.org/2007060254/vol.-Ixiv-2006/n\%C2\%BA-233-enero-abril-2006/pensar-elpensamiento-del-profesorado.html, ISSN: 2174-0909, Revista española de pedagogía, (233), 105-122 (2006)

Judikis, J.C.; C.A. Estrada; M.D. Makuc; W.M. Molina y R.A. Cárcamo, Teorías implícitas sobre el aprendizaje y su relación con las prácticas pedagógicas: Un estudio de caso. En: Selección de investigaciones primer concurso FONIDE: Evidencias para políticas públicas en educación, Mineduc editor. Editorial Gráfica LOM Ltda., Santiago, Chile (2008)

Makuc, M., Teorías implícitas sobre comprensión textual y la competencia lectora de estudiantes de primer año de la Universidad de Magallanes, doi: 10.4067/S0718-07052011000100013, Estudios pedagógicos, 37(1), 237-254 (2011)

Martínez, J.R. Concepción de Aprendizaje, Metacognición y Cambio Conceptual en Estudiantes Universitarios de Psicología. Tesis Doctoral para optar al grado de Doctor de la Universidad de Barcelona, Universidad de Barcelona, Barcelona-España (2004)

Martín, E.M. y otros 6 autores. Proyecto 06/0017/2001. Investigación financiada por la Comunidad de Madrid. Convocatoria de Humanidades (2001)

Martín, E., M. Mateos, P. Martínez, J. Cervi, A. Pecharromán y R. Villalón. Las concepciones de los profesores de educación primaria sobre la enseñanza y el aprendizaje. En J. I. Pozo, N. Scheuer, M. P. Pérez Echeverría, M. Mateos, E. Martín, y M. de la Cruz (Eds.) Nuevas formas de pensar la enseñanza y el aprendizaje, pp. 171187. Graó, Barcelona, España (2006)

Martín, E.M., J.I. Pozo, M.P., Pérez Echeverría, M.M. Mateos y A. Martín, ¿Cómo se representan los profesores el aprendizaje y la enseñanza? De las concepciones a los perfiles docentes. En: La Identidad en Psicología de la Educación: necesidad, utilidad y límites, C. Monereo y J. Pozo editores. Narcea, Madrid, España (2011)

MINEDUC. Informe Comisión sobre Formación Inicial Docente. Documento no oficial, Serie Bicentenario. Santiago, Chile (2005)

$\mathrm{Ng}, \mathrm{W}$; Nicholas H. y Williams, A., School experience influences on preservice teachers' evolving beliefs about effective teaching, doi:10.1016/j.tate.2009.03.010, Teaching and Teacher Education, 26, 278-289 (2010)

OCDE. Revisión de políticas nacionales de educación. OCDE y Ministerio de Educación de Chile. Chile (2004) 
Oliver Vera, C., El valor formativo y las ataduras de las creencias en la formación del profesorado. Aquello que no se ve, pero se percibe en el aula, http://www.aufop.com, ISSN: 1575-0965, Revista Interuniversitaria de Formación del Profesorado, 12(1), 63-75 (2009)

Paukner-Nogués. F., S.V. Sanhueza-Henríquez y V.H. San Martín-Ramírez, Evaluación de la práctica docente en la reforma educacional chilena. Educación y educadores, 15(3), 345-362 (2012)

Pérez Echeverría, M., J. I., Pozo, A. Pecharromán, J. Cervi y P. Martínez, Las concepciones de los profesores de educación secundaria sobre el aprendizaje y la enseñanza. En J. I. Pozo, N. Scheuer, M. P. Pérez Echeverría, M. Mateos, E. Martín, y M. de la Cruz (Eds.) Nuevas formas de pensar la enseñanza y el aprendizaje, pp. 289-304. Graó, Barcelona, España (2006)

Pozo, J.I., N. Scheuer, M.P. Pérez Echeverría, M.M. Mateos, E.A. Martín y M. De la Cruz. Nuevas Formas de Pensar la Enseñanza y el Aprendizaje. Graó, Barcelona-España (2006)

Pozo, J.I. y N. Scheuer, Las concepciones sobre el aprendizaje como teorías implícitas. En: El aprendizaje estratégico, J. Pozo y C. Monereo coordinadores. Santillana, Madrid, España (1999)

Pozo, J.I., Aprendices y maestros: La psicología cognitiva del aprendizaje. Alianza, Madrid, España (2008)

Rodrigo, M.J. y N.T. Correa, Representación y procesos cognitivos: esquemas y modelos mentales. En: Psicología de la educación escolar. C. Coll, J. Palacios y A. Marchessi compiladores. Alianza, Madrid- España (2001)

Rodrigo, M.J., A. Rodríguez y J.E. Marrero, Las teorías implícitas. Una aproximación al conocimiento cotidiano. Visor, Madrid, España (1993)

Rosas, R.R. y C.L. Sebastián, Piaget, Vigotski y Maturana. Constructivismo a tres voces. $2^{2}$ reimpresión. Aique, Buenos Aires, Argentina (2008)

Seebauer, R. The modification of subjective theories with viennese teacher trainees: results from a two-year study between the first and the fourth semester of study, http://www.orbisscholae.cz/archiv/2009/2009_2_05.pdf, Orbis Scholae, 3(2), 63-75, (2009)

Shavelson, R.J. y P.R. Stern, Investigación sobre el pensamiento pedagógico del profesor, sus juicios, decisiones y conductas. En: La enseñanza. Su teoría y su práctica, Sacristán, J. y A. Pérez Gómez editores. Akal, Madrid-España (1989)

Schnotz, W., S. Vosniadou y M. Carretero, Cambio conceptual y educación. Aique, Buenos Aires-Argentina (2006)

van Dinther, M., F. Dochy y M. Segers, M., The contribution of assessment experiences to student teachers' self-efficacy in competence-based education, doi:10.1016/j.tate.2015.02.013, Teaching and Teacher Education, 49, 45-55 (2015)

von Reininghaus, G, P.J. Castro y S. Frisancho, School violence: subjective theories of academic advisory board members from six chilean schools, http://www.scielo.org.ar/scielo.php?pid=S166870272013000200003\&script=sci_arttext, ISSN 1668-7027, Interdisciplinaria, 30(2), 219-234 (2013) 
